\title{
Review paper: A Briefing to Grey Systems Theory
}

\author{
Qiaoxing LI \\ School of Management, Lanzhou University, Lanzhou 730000, China \\ E-mail: liqiaoxing@eyou.com \\ Yi LIN \\ Department of Mathematics, Slippery Rock University, Slippery Rock, PA 16057, USA \\ E-mail: Jeffrey.forrest@sru.edu
}

\begin{abstract}
Grey systems theory, probability theory, fuzzy systems theory and rough set theory are known as four uncertain systems scientific methodologies. After thirty years of development, grey systems theory has formed its own disciplinary framework, whose content includes grey philosophy, grey mathematics, processing and technology systems of grey information, as well as grey systems engineering. However, as a developing area of scientific activities, a lot of theoretical contents are still imperfect and awaiting further studies. The purpose of this paper is to acquaint the readers with the development history, the main content and the major challenges of grey system theory. It is our hope that this paper could play the role of attracting the attention of the international academic community to the theory so that the development of this theory could be further promoted.
\end{abstract}

Keywords uncertainty; poor information; information processing; information technology; development process

\section{Introduction}

Starting when Italian scholars began their study of dice tossing in the 16th century and when people explored stochastic processes at the end of the 19th century, a large number of mathematicians, such as Pascal, de Fermat, Huygens, Bernoulli, de Dimofu, Laplace, Chebyshev, Markov, Lyapunov, etc., had devoted their arduous efforts. At the same time, the appearance of the second law of thermodynamics at around the middle of the 18th century motivated people to seriously consider and summarize the laws that govern the phenomena of randomness. With the diligent works of many scholars over the past several hundred years, the theory of probability, the first science that studies uncertain phenomena, was eventually established. Accompanying the development of industrialization and the global emergence of economic and social activities, the very way of how people explore and understand nature started to shift gradually from the original sole focus on objects of interest themselves to that of fathoming the matters or events of concern through understanding the information gathered from the external environment and to the attempt of controlling the development of the external environment. Consequent to such trend, there have appeared theories like systems, information, and cybernetics. The intertwining economic and social environments, so complicated that they have never

Received November 20, 2013, accepted January 3, 2014 
seen before in history, give rise to many modern day complex scientific problems, which in turn promote the vigorous development of different branches of systems science. These new theories have helped deepen our understandings of the evolution of the physical nature and subjective matters. Due to the complexity of the external environment and the inherent limitation of human ability to acquire information, various types of uncertain phenomena, different of those of stochastic problems, are discovered in the human efforts of understanding and changing the world. These discoveries led to three additional methodologies of uncertain systems developed to deal with the corresponding problems of uncertainty. These methodologies are respectively the fuzzy systems theory, initiated by Zadeh, rough set theory, established by Pawlak, and grey systems theory, proposed by Julong Deng. Presently, together with probability they are jointly known as four systems scientific methods developed for dealing with uncertainties. At their respective starts, fuzzy systems theory, rough set theory and grey systems theory all suffered from various degrees of difficulties. For instance, fuzzy systems theory had been questioned by many for a long period of time. These scholars believed that with the tool of probability there was no longer any need for fuzzy systems theory (see [1]). For the theory of rough sets, it did not capture the attention of the world of learning many years after Pawlak introduced it. Similarly, along with the development of grey systems theory, it was also severely challenged by various scholars. In order to further the understanding and development of grey systems theory at a more global level, this article will briefly describe the development history and main contents of this new theory.

In 1982, Julong Deng published in Systems \& Control Letters "The Control Problems of Grey Systems", signaling the birth of grey systems theory. As soon as this theory initially appeared, it has caught the attention of the world of learning; especially it has been actively supported by scientific theorists and practitioners of China. With high levels of enthusiasm a great number of scholars explored grey systems theoretically and applied the new-found knowledge in various conventional disciplines. And since Junzheng Chen published two papers respectively in 1988 and 1989 in "Systems Engineering — Theory \& Practice" criticizing this new theory, grey systems theory has been questioned and challenged by some scholars over the past twenty some years, causing the withdrawal of some individual scholars from the early research effort of grey systems theory. Although grey systems theory has been criticized over the years by various individuals, there have been good numbers of scholars who continued their works on grey systems and produced $\mathrm{PhD}$ graduates specializing in this area. It is due to these scholars and the addition of the new scientific bloods that the studies on grey systems have been carried onto higher levels. With nearly thirty years of uninterrupted development, grey systems theory has developed its theoretical framework and an array of successful applications in many scientific fields. Currently, scholars from many countries and geographical regions, such as China, England, USA, Germany, Japan, Australia, Canada, Russia, Taiwan, Hong Kong, etc., have joined in the theoretical and applied works of grey systems. The Journal of Grey Systems, launched in 1989 in England, has been indexed in Science Abstracts (SA), Mathematical Review (MR), Mathematics Abstracts (MA), DIALOG, INSPEC, and Science Citation Index Expanded (SCI). Another periodical named "Journal of Grey Systems", published in English in Taiwan, has also been closely followed by scholars from around the world. In 
2011, Emerald Group of Publishing Limited launched yet another professional periodical "Grey Systems: Theory and Applications". Additionally, works on grey systems have been accepted and published by over 1,000 different publications from around the world, including Applied Mathematical Modelling, Journal of Mathematical Analysis and Applications, Applied Mathematics and Computation, Expert Systems with Applications, etc. Proceedings of American Association for Computing Machinery and Kybernetes: The International Journal of Cybernetics, Systems and Management Science have published special issues on grey systems. In recently years, Chinese Journal Full-Text Database collects more than 1,000 papers on works related to grey systems annually. EI indexes about 700 grey-system papers a year, and SCI and SSCI also index over 200 grey-system related papers each year. Over one hundred universities from across the world, including Huazhong University of Science and Technology (China), Nanjing university of Aeronautics and Astronautics (China), University of Maryland (USA), Toyohashi University (Japan), Kanagawa University (Japan), Viena University of Economics (Austria), French Space Navigation Center (France), National Central University (Taiwan), Cheng Kung University (Taiwan), Tatung University (Taiwan), etc., have offered courses in grey systems theory and recruited and produced graduates specializing in relevant directions. Prestigious publishers, such as Science Press (China), Press of Huazhong University of Science and Technology, Science and Technology Publishing House (China), Springer-Verlag (Germany, England), CRC Press (USA), etc., have published over 100 different volumes on grey systems. Scholars from conventionally disciplines have developed using grey systems theory a large group of hybrid new studies, such as grey hydrology, grey geology, grey plant thremmatology, grey philosophy, grey input-output analysis, grey complex networks, grey extenics, and so on. As of this writing, grey systems theory has been successfully applied to resolving many difficult problems appearing in daily lives, works, and scientific research in a wide range of scientific disciplines, including industry, agriculture, sociology, economics, energy, transportation, petroleum, geology, water conservation, meteorology, ecology, environment, medicine, education, sports, military, law, finance, and others (see [2,3]). For instance, Huang et al. (see [4]) processed the data of grey image compression technology; Xu et al. (see [5]) predicted petroleum and natural gas fields in Tarim Basin in Xingjiang; Lin and Valencia (see [6]) employed grey methodology to their study of migration of people; etc. Many important international conferences, such as International Conference on Modeling of Unascertained Systems, International Conference on Systems Prediction and Control, Workshops of the International Institute for General Systems Studies, Congress of the World Organization of Cybernetics and Systems, International Conference of Computer Science and Industrial Engineering, International Conferences of IEEE System, Man and Cybernetics, etc., have listed grey systems theory as a special topic area. In 2007, IEEE established a technical committee of grey systems and has held international events biannually ever since. This IEEE committee has played an active role of promoting grey systems research. Liu and Lin (see [7]) provides a comprehensive description for the roles and position of grey systems theory in the development of science.

Although the research of grey systems has established some very important results, its theoretical framework is still in need of further completion. As of this writing, the main points of grey systems theory are still debated. For instance, what are the cognitive differences and 
similarities between grey systems theory and probability theory, fuzzy systems theory, and rough set theory? What are the technical differences and similarities between the specific ways of handling information between grey systems theory and other more traditional methods? What are the differences and connections between grey $\operatorname{GM}(1,1)$ model system and grey functions? Etc. Additionally theoretical results of grey systems have been mainly published in Chinese journals, while those works published outside China are mainly applied in nature. To promote further development of grey systems theory, to stimulate international exchanges of ideas, and to correctly employ probability theory, fuzzy systems theory, rough set theory, and grey systems theory to resolve the widely existing problems involving uncertainties in the realistic world, in the rest of this paper, we will briefly introduce the basic concepts and main results of grey systems theory.

\section{Main contents of grey systems theory}

In control theory, scholars often use colors to describe the degree of information certainty. When the information of a system is known with complete certainty, the system is seen as white. On the other extreme, if the information of a system is completely unknown, the system is referred to as black. So, systems with partially known and partially unknown information are known as grey; and phenomena with partially known and partially unknown information are known as those of poor information. The characteristic of poor information is exactly the grayness studied in grey systems theory. Due to the involved systems' complexities, it is often the case that human capability of obtaining the relevant information seems to be quite limited. Therefore, grey phenomena exist universally in the physical world. Correspondingly grey systems theory provides a scientific methodology of uncertain systems for dealing with phenomena of poor information widely existing in the material world. Because of the effect of poor information, the researcher cannot generally obtain the true values of the objects of concern other than the ranges of these values (see $[8,9]$ ). Speaking philosophically, the true values represent certain essential aspects or connotation of the phenomena of concern so that poor information causes uncertainties in the recognition of the connotations of the matter. The concept of grey hazy sets is the set theoretic foundation of grey systems theory, while grey mathematics is the mathematical foundation of grey systems theory. Grey numbers, the basic mathematical elements of grey systems theory, are defined within the framework of grey hazy sets. Through 30 years of development, the fundamental theoretical framework of grey systems theory has been established, containing mainly (for brief introduction in this regards, see [10, 11]: The philosophical system developed on the axioms of uncertainty and grey cognitive principles; The mathematical system generated around the core of grey hazy sets, grey operations and covered operations; A system of methods for dealing with information using grey incidence analysis, grey sequence generations, and grey GM(1,1) model; A system of techniques developed for evaluation, prediction, decision-making, control and optimization (both of these method and technique systems together are known as the technology for processing grey information); A system of applications in grey systems engineering; etc.

Various thoughts of grey systems theory have germinated from the grey philosophical system, which represents an elaboration of this systems science at the philosophical height. Under 
the guidance of grey philosophy, the system of grey mathematics constitutes a system of operations constructed in parallel to the classical mathematics in terms of formal logic. In the technology of processing grey information, grey incidence analysis and grey evaluation stand for two different applications of the same underlying method, where grey evaluation mainly includes grey statistical evaluation, grey cluster evaluation, etc. Grey sequence generations are actualized through applications of sequence operators, which include buffer operators (either strengthening or weakening), mean-generation operators, step-ratio generation operators, accumulation and inverse accumulation generation operators, etc. Grey models are approximate differential equation models useful for modeling such small samples of data as of only 4 data points. The methods of grey prediction include those for sequence prediction, interval prediction, systems prediction, etc. Grey decision-making covers grey-target decision, grey incidence decision, grey situation decision, grey layered decision, etc. Grey control includes incidence control, $\operatorname{GM}(1,1)$ control, and others. The techniques of grey optimization include grey linear programming, grey nonlinear programming, grey integer programming, grey dynamic programming, etc. Grey systems engineering contains grey hydrology, grey input-output analysis, etc. In the following sections, we will one by one outline the main contents of these parts.

\section{The system of grey philosophy}

This system contains the following main axioms and cognitive principles of uncertainty. Grey systems theory focuses on the study of such uncertain systems with partially known and partially unknown information that matters of the characteristics of poor information are seen as its research subjects. It attempts to understand the laws of development of things by utilizing the partially known information, while revealing a correct description, effective monitoring and control of the behaviors of systems and the relevant laws of evolution. Li and Lin (see [8]) summarized the axiomatic system of grey systems theory as follows:

Axiom 1 The connotation of each objective matter or thing is unique.

Connotation reflects a certain essential aspect of the underlying objective matter or thing, and represents an objective existence. At a fixed time moment, chosen location, and an invariant condition, the connotation of the underlying matter or thing is uniquely determined. Each process of exploration of the connotation constitutes a problem of cognition. The research subjects of grey systems theory are about the cognitive problems of connotations of various objective matters; and each cognitive process is completed within the complex system that contains the specific objective matter. At the same time, the system does not contain the complete information for one to fully fathom the connotation of the matter. That is, cognition of connotations possesses the characteristics of poor information.

Axiom 2 The connotation of each objective matter or thing possesses relativity.

Grey systems theory recognizes the cognitive relativity of connotations. Such cognitive relativity can be illustrated from three different angles. First, speaking generally, the connotation of matters represents only the correct cognition within a certain scope; secondly, in terms of depth, each recognized connotation is only an approximate understating of the underlying objective matter and relevant laws and awaits deepening explanation; and thirdly, in terms of processes, connotations of matters stand only for the correct cognition for a certain time period 
and need to evolve further (see [12]). Hence, each cognitive connotation of objective matters is relative. Only by applying appropriate methods of investigation, one could possibly obtain and effectively deal with the information that truthfully reflects the connotation of the underlying matter, leading to relative cognition of the connotation.

Axiom 3 Information exists objectively and represents the unification of commonality and individuality.

Information stands for another objective reality that co-exists side by side along with mass and energy in modern science. Each system is generally composed of mass, energy, information, and others. The concept of information can be understood either generally or specifically. The kind of information grey systems theory deals with belongs to the general category. There are two basic significance of general information. At the level of materialism, information represents the state and form of motion of materials; and at the level of epistemology, information stands for a certain expression or reflection of the state and form of motion of the materials, as felt by the observer (see [12]). News, signals, data, intelligence, knowledge, etc., are some of the forms of recording information and cannot be seen as the same as the information. At the same time, information also stands for the unification of commonality and individuality, where commonality means the indifference of information. A piece of information is the information about a certain aspect of the connotation of the matter, and also the information of the same connotation (respectively, a different aspect of the connotation of the same matter or a different connotation of a different matter) of another matter (respectively, a different aspect of the connotation of the same matter or a different connotation of a different matter). The individuality of information represents the information's specific effects in terms of the specified matter and its specified connotation. The same piece of information has different effects for either different connotations or the same observer but in different states.

These three axioms together constitute the axiomatic system of grey systems theory. Within this system, Li, Lin and Deng (see $[8,13]$ ) derived the following fundamental principles:

1) Principle of Acquiescence: If no reason exists to justify the falsity of Proposition A, then acquiesce the truthfulness of Proposition A.

2) Principle of Informational Differences: Different pieces of information must be different; even the same piece of information has to be different when analyzed from different angles. Differences also stand for information.

3) Principle of Recognition Base: Information is the foundation on which one recognizes and understands (nature). Recognition has to have basis.

4) Principle of New Information Priority: New pieces of information possess more weights than old pieces.

5) Principle of Information Uncertainty: Information processing can either increase or decrease the adequacy of cognition.

6) Principle of Absolute Greyness: Incompleteness of information is absolute. That is, human cognition is forever grey.

On the basis of these principles of grey cognition, Deng (see [13]) provided an initial discussion of elementary models of grey cognition, beyond which there are still many open problems in the system of grey philosophy that await further elaboration. For example, one needs to 
address how he can distinguish the four uncertain systems theories from the point of view of epistemology, while pointing out their connections; to further detail the illustrations and properties of grey axiomatic system and the principles of cognition; to explore the laws that govern human cognition; the concept of grey information, etc. In particular, there is a desperate need to epistemologically distinguish the four theories of uncertainties so that fuzzy and grey systems and rough set theories can righteously enjoy their individual deserving attention of the scientific community.

\section{The system of grey mathematics}

This is mainly a computational system centered on grey and covered operations, developed, under the guidance of grey philosophy, on the basis of grey hazy sets along side with the rules of formal logic reasoning of the conventional mathematics (see [8]). The concept of grey hazy sets is the set-theoretic basis of grey systems theory, which includes four types of numbers: grey numbers, number-covered set, whitened numbers, and the only potential true numbers. A grey number is an uncertain quantity, a quantitative expression of the connotation of the matter of concern, and a basic element of grey mathematics. Because of the relativity of the matter's cognitive connotation and the characteristic of poor information, one can only obtain a covered set of the connotation, whose quantitative expression is the number-covered set of the grey number. A whitened number can be any particular value within the number-covered set; it represents an approximation of the true value of the grey number. The true value of the grey number has to be within the number-covered set. Because of the uniqueness of connotations, the true value of any grey number exists uniquely, known as the only potential true number. When the connotation of a matter is completely known, the grey uncertain number turns into a real number. That is why grey mathematics takes the same forms of operation of the conventional mathematics. In particular, other than such basic operations as addition, subtraction, multiplication, and division, there are also limit, differentiation, and integration operations. At the same time, grey mathematics possesses its own additional rules and particular forms of operation. There are four main forms of operation in grey mathematics: grey operations, covered operations, whitened operations, and operations of only potential true numbers. Here, operations between grey numbers are known as grey number operations, those between number-covered sets as number-covered operations, those between only potential true numbers as potential true operations, and those between whitened values as whitened operations. Grey and only potential true operations are the essence and stand for interactions of connotations. Covered and whitened operations represent interactions of forms and of extensions. Covered operations represent some of the holistic approximate computations. Whitened operations are identical to the approximate calculations of the definite form of the conventional mathematics. What is important in grey mathematics is how to perform grey and covered operations. Because grey operations are quantitative with the characteristics of uncertainty, they need to be analyzed quantitatively. Covered operations represent some of the forms of operation with evolutionary characteristics developed on the basis of grey operations. To better illustrate grey numbers, grey and number-covered operations, etc., let us use an example to briefly explain the basic results and rules of these two kinds of operations (see $[8,12-16]$ ). 
Example 1 There are two logs of different but roughly equal lengths, whose specific values are unknown. So, the lengths of these logs are grey numbers, donated respectively by $\otimes_{1}$ and $\otimes_{2}$. With additional given information, they can be estimated that the lengths are between 10 and 10.1 meters. That is, the number-covered sets of both $\otimes_{1}$ and $\otimes_{2}$ are the interval $[10,10.1]$, indicating that the only potential true numbers of $\otimes_{1}$ and $\otimes_{2}$ fall within $[10,10.1]$. Because these logs are of different lengths, the only potential true numbers of $\otimes_{1}$ and $\otimes_{2}$ are different. Even so, 10.01 is a whitened value for both $\otimes_{1}$ and $\otimes_{2}$.

When expressing the number-covered set of a grey number, it is important to point out which grey number is concerned with, because a grey number has different number-covered sets, while the same number set can be number-covered sets of many different grey numbers.

In grey mathematics, grey number operations satisfy the following results (for proofs, see $[13,15])$ :

Theorem 1 For any grey number $\otimes$, the equation $\otimes-\otimes=0$ holds.

Theorem 2 Assume that $d_{1}^{\circ}$ and $d_{2}^{\circ}$ are respectively the only potential true numbers of $\otimes_{1}$ and $\otimes_{2}$. Then $\otimes_{1}=\otimes_{2} \Leftrightarrow d_{1}^{\circ}=d_{2}^{\circ}$.

Theorem 3 For given grey numbers $\otimes_{1}, \otimes_{2}$ and $\otimes_{3}$, and $\circ \in\{+,-, \times \times\}$, grey number operation o satisfies the following rules:

1) Commutativity of addition and multiplication: $\otimes_{1}+\otimes_{2}=\otimes_{2}+\otimes_{1}$ and $\otimes_{1} \times \otimes_{2}=\otimes_{2} \times \otimes_{1}$;

2) Associativity of addition and multiplication: $\left(\otimes_{1}+\otimes_{2}\right)+\otimes_{3}=\otimes_{1}+\left(\otimes_{2}+\otimes_{3}\right)$ and $\left(\otimes_{1} \times \otimes_{2}\right) \times \otimes_{3}=\otimes_{1} \times\left(\otimes_{2} \times \otimes_{3}\right) ;$

3) Distributivity: $\left.\otimes_{1} \times\left(\otimes_{2}+\otimes_{3}\right)=\otimes_{1} \times \otimes_{2}+\otimes_{1} \times \otimes_{3}\right)$.

In general, a number-covered set $D$ of grey number $\otimes$ takes one of the following two forms:

1) If $D$ is a discrete set of numbers, then $D$ is known as a discrete number-covered set of the grey number $\otimes$;

2) If $D$ is a continuous set or an interval of numbers, then $D$ is known as a continuous number-covered set of $\otimes$.

The particular forms of covered operations are as follows:

Definition 1 Assume that discrete number-covered sets of grey numbers $\otimes_{i}$ and $\otimes_{j}$ are respectively $D_{i}=\left\{d_{i k} \mid k=1,2, \cdots, n\right\}$ and $D_{j}=\left\{d_{j l} \mid l=1,2, \cdots, m\right\}$. Let $\otimes_{i j}=\otimes_{i} \circ \otimes_{j}$ and $D_{i j}=D_{i} \circ D_{j}$, where $\circ \in\{+,-, \times, \div\}$, then a discrete number-covered set $D_{i j}$ of the grey number $\otimes_{i j}$ is given by

i) $D_{i j}=D_{i}+D_{j}=\left\{d_{i k}+d_{j l} \mid k=1,2, \cdots, n ; l=1,2, \cdots, m\right\}$;

ii) $D_{i j}=D_{i}-D_{j}=\left\{d_{i k}-d_{j l} \mid k=1,2, \cdots, n ; l=1,2, \cdots, m\right\}$;

iii) $D_{i j}=D_{i} \times D_{j}=\left\{d_{i k} \times d_{j l} \mid k=1, \cdots, n ; l=1,2, \cdots, m\right\}$; and

iv) $D_{i j}=D_{i} \div D_{j}=\left\{d_{i k} \div d_{j l} \mid k=1,2, \cdots, n ; l=1,2, \cdots, m\right\}$, where $0 \bar{\in} D_{j}$.

Definition 2 Assume that continuous number-covered sets of grey numbers $\otimes_{i}$ and $\otimes_{j}$ are respectively $D_{i}=\left[a_{i}, b_{i}\right]$ and $D_{j}=\left[a_{j}, b_{j}\right]$. Let $\otimes_{i j}=\otimes_{i} \circ \otimes_{j}$ and $D_{i j}=D_{i} \circ D_{j}$, where $\circ \in\{+,-, \times, \div\}$, then a continuous number-covered set $D_{i j}$ of the grey number $\otimes_{i j}$ is given by

i) $D_{i j}=D_{i}+D_{j}=\left[a_{i}+a_{j}, b_{i}+b_{j}\right]$;

ii) $D_{i j}=D_{i}-D_{j}=\left[a_{i}-b_{j}, b_{i}-a_{j}\right]$;

iii) $D_{i j}=D_{i} \times D_{j}=\left[\min \left\{a_{i} a_{j}, a_{i} b_{j}, b_{i} a_{j}, b_{i} b_{j}\right\}\right]$; and 
iv) $D_{i j}=D_{i} \div D_{j}=\left[\min \left\{a_{i} / a_{j}, a_{i} / b_{j}, b_{i} / a_{j}, b_{i} / b_{j}\right\}\right]$, where $0 \bar{\in} D_{j}$.

Definition 3 Assume that a discrete number-covered set of grey number $\otimes_{i}$ is $D_{i}=\left\{d_{i k}\right.$ $k=1,2, \cdots, n\}$, and a continuous number-covered set of grey number $\otimes_{j}$ is $D_{j}=\left[a_{j}, b_{j}\right]$. Let $\otimes_{i j}=\otimes_{i} \circ \otimes_{j}$ and $D_{i j}=D_{i} \circ D_{j}$, where $\circ \in\{+,-, \times, \div\}$, then a number-covered set $D_{i j}$ of the grey number $\otimes_{i j}$ is given as follows:

i) $D_{i j}=D_{i}+D_{j}=\cup_{k=1}^{n}\left[d_{i k}+a_{j}, d_{i k}+b_{j}\right]$;

ii) $D_{i j}=D_{i}-D_{j}=\cup_{k=1}^{n}\left[d_{i k}-b_{j}, d_{i k}-a_{j}\right]$;

iii) $D_{i j}=D_{i} \times D_{j}=\cup_{k=1}^{n}\left[\min \left\{d_{i k} a_{j}, d_{i k} b_{j}\right\}, \max \left\{d_{i k} a_{j}, d_{i k} b_{j}\right\}\right]$; and

iv) $D_{i j}=D_{i} \div D_{j}=\cup_{k=1}^{n}\left[\min \left\{d_{i k} / a_{j}, d_{i k} / b_{j}\right\}, \max \left\{d_{i k} / a_{j}, d_{i k} / b_{j}\right\}\right]$, where $0 \bar{\in} D_{j}$.

Note: Grey numbers also have other forms of covered sets and relevant operations. For details, see [14-16]. Additionally, operations of number-covered sets merely stand for combinations of sets of numbers, while operations of grey numbers produce the results of quantitative analysis. According to the principle of information uncertainty, in practice one should first perform grey number operations, then relevant number-covered operations, then followed by qualitative analysis.

Example 2 Let $D_{1}=\{0,2,9\}$ and $D_{2}=[-3,2]$ be respectively a number-covered set of grey numbers of $\otimes_{1}$ and $\otimes_{2}$. Compute the corresponding number-covered set of the grey number $\left(\otimes_{1}\right)^{2}-\otimes_{1}\left(\otimes_{1}+\otimes_{2}\right)$.

Solution Firstly perform the grey number operations. Because of $\left(\otimes_{1}\right)^{2}-\otimes_{1}\left(\otimes_{1}+\otimes_{2}\right)=$ $-\otimes_{1} \otimes_{2}$, it follows that the desired number-covered set is

$$
D=-\{0,2,9\} \times[-3,2]=\{-9,-2,0\} \times[-3,2]=[-18,27] \cup[-4,6] \cup\{0\}=[-18,27] .
$$

It is worth noticing that if we did not perform the grey number operations first, but instead we directly substituted the number-covered sets of $\otimes_{1}$ and $\otimes_{2}$ into $\left(\otimes_{1}\right)^{2}-\otimes_{1}\left(\otimes_{1}+\otimes_{2}\right)$, then the consequent covered operations lead to $D=[-95,-50] \cup[-36,31] \cup[45,108]$, which is evidently a worse number-covered set.

Definition 4 If a real matrix A contains at least one grey entry, then $A$ is known a grey matrix, denoted $A(\otimes)$.

Definition 5 For grey matrix $A(\otimes)=\left(\otimes_{i j}\right)_{m \times n}$, let $D_{i j}$ be a number-covered set of $\otimes_{i j}$ $(i=1,2, \cdots, m ; j=1,2, \cdots, n)$, then $A(D)=\left(D_{i j}\right)_{m \times n}$ is known as a matrix-covered set of $A(\otimes)$.

Accordingly, one can define the concepts of whitened and only potential true matrices of grey matrices. Then between grey matrices can be defined grey matrix operations; and between matrix-covered sets can be defined matrix-covered operations. Similarly, whitened and only potential true matrix operations can be introduced. In particular, the following two definitions provide the specific details of matrix-covered operations:

Definition 6 Assume that $A(D)=\left(D_{i j}\right)_{m \times n}$ and $B(D)=\left(D_{i j}^{\prime}\right)_{m \times n}$ are respectively matrix-covered sets of grey matrices $A(\otimes)=\left(\otimes_{i j}\right)_{m \times n}$ and $B(\otimes)=\left(\otimes_{i j}^{\prime}\right)_{m \times n}$. For $\circ \in\{+,-\}$, let $A(D) \circ B(D)$ be a matrix-covered set of $A(\otimes) \circ B(\otimes)$, then $A(D)+B(D)=\left(D_{i j}+D_{i j}^{\prime}\right)_{m \times n}$ and $A(D)-B(D)=\left(D_{i j}-D_{i j}^{\prime}\right)_{m \times n}$, where $D_{i j}+D_{i j}^{\prime}$ and $D_{i j}-D_{i j}^{\prime}(i=1,2, \cdots, m ; j=1,2, \cdots, n)$ are respectively the addition and subtraction operations of number-covered sets.

Definition 7 Assume that $A(D)=\left(D_{i j}\right)_{m \times n}$ and $B(D)=\left(D_{i j}^{\prime}\right)_{m \times n}$ are respectively matrix-covered sets of grey matrices $A(\otimes)=\left(\otimes_{i j}\right)_{m \times n}$ and $B(\otimes)=\left(\otimes_{i j}^{\prime}\right)_{m \times n}$. Then $A(D) \times$ 
$B(D)$ is the matrix-covered set of $A(\otimes) \times B(\otimes)$ defined by $A(D) \times B(D)=\left(\sum_{k=1}^{l}\left(D_{i k} \times\right.\right.$ $\left.\left.D_{k j}^{\prime}\right)\right)_{m \times n}$, where $D_{i k} \times D_{k j}^{\prime}(i=1,2, \cdots, m ; j=1,2, \cdots, n)$ stands for the multiplication operation of number-covered sets.

Definition 8 For a real-valued function $y=f\left(x_{1}, x_{2}, \cdots, x_{n} ; a_{1}, a_{2}, \cdots, a_{m}\right)$, where $x_{1}, x_{2}$, $\cdots, x_{n}$ are variables and $a_{1}, a_{2}, \cdots, a_{m}$ constant parameters, if at least one of the parameters is grey, then the function is known as a grey function, denoted $y(\otimes)=f\left(x_{1}, x_{2}, \cdots, x_{n} ; a_{1}(\otimes), a_{2}(\otimes)\right.$, $\left.\cdots, a_{m}(\otimes)\right)$, where $a_{1}(\otimes), a_{2}(\otimes), \cdots, a_{m}(\otimes)$ are referred to as grey parameters.

Definition 9 Assume that $[y]$ and $\left[a_{i}\right]$ are respectively number-covered sets of grey numbers $y(\otimes)$ and $a_{i}(\otimes)(i=1,2, \cdots, m)$. Then the function-covered set $[y]$ of the grey function $y(\otimes)=$ $f\left(x_{1}, x_{2}, \cdots, x_{n} ; a_{1}(\otimes), a_{2}(\otimes), \cdots, a_{m}(\otimes)\right)$ is given by $[y]=f\left(x_{1}, x_{2}, \cdots, x_{n} ;\left[a_{1}\right],\left[a_{2}\right], \cdots,\left[a_{m}\right]\right)$.

Accordingly, the concepts of whitened and only potential true functions can be established; and grey function, function-covered, whitened function, and potential true function operations can be defined.

In the system of grey mathematics, both grey and covered operations represent the core. One of the problems grey mathematics attempts to address is to establish computational formulas for various covered operations. Specifically, for instance, for the grey sine function $y(\otimes)=\sin (a(\otimes)+b(\otimes) x)$, when the number-covered sets $[a]$ and $[b]$ of $a(\otimes)$ and $b(\otimes)$ are given, how can one establish a computational formula to obtain such a number-covered set $[y]$ of $y(\otimes)$ that $[y]$ contains as small disturbance as possible. Although the research of grey mathematics has established some significant results, many fundamental aspects, such as the properties and operations of grey hazy sets, grey number analysis, rules of grey and covered operations of grey matrices and those of grey functions, still await further and deepened investigation. Additionally, a very important and urgent area of work is the comparison between grey mathematics and other branches of uncertainty mathematics.

A historical note: A similar concept in notion, named interval analysis, was originated independently by Sunaga and Moore (see [17-18]) and developed ever since the 1950s by a score of mathematicians as an approach to putting bounds on rounding errors and measurement errors in mathematical computations. It represents a numerical quantity as an interval within which the true value of the quantity lies. This end is exactly the same as the concept of grey interval numbers. The arithmetic operations, such as,,$+- \times, \div$, of interval numbers are defined exactly the same as those of the interval grey numbers (see [19]). As other scientific concepts, the idea of using intervals to estimate numbers has appeared throughout the history in different names. For example, Archimedes estimated the value of using the interval $[223 / 71$, 22/7] in the third century BC. In 1931, Rosalind Cicely Young of University of Cambridge published a paper, entitled "algebra of many-valued quantities", that gives rules for calculating with intervals and other sets of real numbers (see [20]). Arithmetic work on range numbers to improve reliability of digital systems were then published in a textbook on linear algebra by Dwyer (see [21]) of University of Michigan, where intervals were used to measure rounding errors associated with floating-point numbers. Warmus (see [22]) established formulas for interval arithmetic operations in both endpoint and midpoint-radius formulations, and the definition of the centered outer multiplication. He then developed an interval space diagram using midpointradius coordinates in [23]. Sunaga (see [17]) contained many of the same ideas of interval 
analysis as later (Moore, 1962) developed in his PhD dissertation. As of this writing, most of the modern literature on interval analysis traces back to [24] as the official start of this area of research. One of the merits of interval analysis is that starting with a set of simple principles it provides a general method for automated error analysis, not just errors resulting from rounding. In the following twenty years, groups of German scholars, headed by Alefeld and Kulisch (see $[25,26]$ ), carried out pioneering works. In particular, among others, Nickel (see [27]) explored more effective implementations; and Neumaier (see [28]) improved containment procedures for the solution set of systems of equations. Hansen (see [29-32]) dealt with interval extensions for linear equations and then provided crucial contributions to global optimization. Nickel (see [27]) published in interval analysis. Ratschek and Rokne (see [33]) developed the branch and bound methods, which till then had been only applied to integer values, by using intervals to provide applications for continuous values. Hansen and Walster (see [34]) studied reliable solution of linear systems and other topics. Since the 1990s, Reliable Computing, currently a Springer journal dedicated to the reliability of computer-aided computations, has been published.

The purpose of this note is to point to the relevant literature and caution the reader that grey systems theory and interval analysis are two completely different areas of research. Based on what is presented in this section, it can be seen that interval analysis can be correctly placed inside grey mathematics, while the research of grey systems is way more than accurate computation. Indeed it is more about dealing with a kind of uncertainty the scientific world has been experiencing since the dawn of the human history. As clearly pointed out by Lin and OuYang (see [35]), other than the design and production of durable goods, where modern science finds its stronghold, a great deal of human endeavors is about predicting the future, where the core concept is structures instead of numerical computations. That is, grey systems theory has more wide-range impact than interval analysis, while studies of interval analysis can and will help build the mathematical foundation of grey systems theory.

\section{Processing and technology of grey information}

The information processing system of grey systems theory mainly contains grey incidence analysis, grey sequence generation, grey modeling, etc. Grey incidence analysis investigates degrees of association between systems' characteristic and other factors based on the similarities observed in the development trends of the factors. This analysis can be employed with a few data points (generally not fewer than 3 ) and does not require the data to satisfy any pre-determined distribution. The main task of such analysis is to determine the dominant and secondary factors that influence the development of the system of interest by using the system's observed behavioral data. Currently, there exist many computational formulas of different forms for the calculation of degrees of grey incidence. For instance, Li (see [14]) introduced the concept of point-slope degree of grey incidence based on the relative distances between the initial points of data sequences and the changes in the slopes of the corresponding curves.

Definition 10 Assume that $X_{0}=\left(x_{0}(1), x_{0}(2), \cdots, x_{0}(n)\right)$ and $X_{i}=\left(x_{i}(1), x_{i}(2), \cdots, x_{i}(n)\right)$ are respectively characteristic sequences and the $i$ th $(i=1,2, \cdots, m)$ factor sequence of a sys- 
tem of concern. Then

$$
\varepsilon_{0 i}=\alpha \frac{1}{n-1} \sum_{k=1}^{n-1} \frac{1}{1+\left|\Delta x_{0}(k)-\Delta x_{i}(k)\right|}+\beta \frac{1+\left|x_{0}(1)\right|+\left|x_{i}(1)\right|}{1+\left|x_{0}(1)\right|+\left|x_{i}(1)\right|+\left|x_{0}(1)-x_{i}(1)\right|}
$$

is the point-slope degree of grey incidence of $X_{0}$ and $X_{i}$, where $\alpha$ and $\beta$ are respectively referred to as the slope and initial-point coefficients satisfying $\alpha, \beta \geq 0$ and $\alpha+\beta=1, \Delta x_{0}(k)=$ $x_{0}(k+1)-x_{0}(k)$ and $\Delta x_{i}(k)=x_{i}(k+1)-x_{i}(k)$ respectively represent the slope of the curves of $X_{0}$ and $X_{i}$ between points $k$ and $k+1, k=1,2, \cdots, n-1 ; i=1,2, \cdots, m$.

By grey sequence generation, it means to uncover the laws that govern the development of the event or system of interest by processing the original data, by transforming the seemingly chaotic data into those with clearly observable patterns so that an overall development trend could be discovered. The generation of grey sequences is done mainly through buffer operators (either weakening or strengthening), mean-generation operators, step-ratio generation operators, accumulation and inverse accumulation operators, etc. In order not to make this paper too long, we will only focus on the presentation of accumulation and inverse accumulation generations (see [19]).

Definition 11 Assume that the original data sequence is $X^{0}=\left(x_{1}^{0}, x_{2}^{0}, \cdots, x_{n}^{0}\right)$ and the sequence obtained from using grey sequence generation is $X^{e}=\left(x_{1}^{e}, x_{2}^{e}, \cdots, x_{n}^{e}\right)$. Then

1) The computational formula of accumulation generation is: $x_{k}^{e}=\left(x_{1}^{0}, x_{2}^{0}, \cdots, x_{k}^{0}\right)(k=$ $1,2, \cdots, n)$; and

2) The inverse accumulation is calculated by $x_{k}^{e}=x_{k}^{0}-x_{k-1}^{0}(k=1,2, \cdots, n)$, where $x_{1}^{e}=x_{1}^{0}$.

For example, if the original sequence is $X=(2,6,3,6,8)$, then the accumulation generated sequence is $X^{e}=(2,8,11,17,25)$, and the inverse accumulation generated sequence is $X^{e}=$ $(2,4,-3,3,2)$.

Additionally, based on the fact that the accumulation and release of energies generally comply with the law of exponentiality, grey systems theory establishes a series of exponential GM models, which can be and have been widely applied in various real-life situations. By employing grey models, one can analyze which system's factors are controllable, which are uncontrollable, and which need to be restricted, so that correct decisions can be quickly made for the system. In the following, let us look at the basic formula of grey models (see [13, 19]).

Definition 12 Assume that $X^{0}=\left(x_{1}^{0}, x_{2}^{0}, \cdots, x_{n}^{0}\right)$ is a non-negative sequence, that is $x_{i}^{0} \geq 0(i=1,2, \cdots, n)$, the accumulation generated sequence $X^{e}=\left(x_{1}^{e}, x_{2}^{e}, \cdots, x_{n}^{e}\right)$ of $X^{0}$, and the mean-generated sequence $Z^{e}=\left(z_{2}^{e}, z_{3}^{e}, \cdots, z_{n}^{e}\right)$ of $X^{e}$, where $z_{k}^{e}=\left(x_{k}^{e}+x_{k-1}^{e}\right) / 2$ $(k=2,3, \cdots, n)$. Then $x_{k}^{0}+a z_{k}^{e}=b$ is referred to as the basic grey model, denoted $\operatorname{GM}(1,1)$.

As of this writing, the research of grey incidence analysis, grey sequence generation, and grey models have led to a great amount of results, produced numerous computational formulas. For details, please consult with the relevant references, such as [9, 35].

Other than methodologies of dealing with information, grey information theory also contains some relevant techniques. Grey information technology mainly investigates methods of how to make use of information, including grey evaluation, grey prediction, grey decision-making, grey control, grey optimization, etc. Grey evaluation consists mainly of grey cluster evaluation and grey statistical evaluation. Grey cluster evaluation includes grey incidence clustering, grey 
whitenization weight function clustering, etc. In the following, let us look at the evaluation method of grey incidence clustering (see $[13,19]$ ). As for the other relevant contents of grey technology, please consult with $[13,19,36]$.

Grey incidence clustering is mainly applied to group factors with certain specified characteristics so that the study of complicated systems can be simplified. Through grey incidence clustering, one can make use of one factor to represent several other closely related factors so that the available information of these factors are not severely damaged; at the same time, the amount of data collection can be greatly reduced [13].

Definition 13 Assume that there are $n$ subjects to be observed, where $m$ characteristic data are collected from each subject, so that the data sequences $X_{i}=\left(x_{i}(1), x_{i}(2), \cdots, x_{i}(n)\right)$ $(i=1,2, \cdots, m)$ are obtained.

Compute the degree $\varepsilon_{i j}$ of grey incidence, $1 \leq i, j \leq m$, between the $i$ th and $j$ th characteristics $X_{i}$ and $X_{j}$ For an arbitrarily chosen constant $r, 0 \leq r \leq 1$, if $\varepsilon_{i j} \geq r$, then the characteristics $X_{i}$ and $X_{j}$ are seen as of the same $r$-class; otherwise they are considered different characteristics.

Information is the soul of grey systems theory. It permeates the entire process of understanding and resolving grey systems problems. The research on the grey-information handling system and grey-information technology system plays an indispensable role in the development of the theory and methodology of grey systems. However, the main results of these two systems are about methods of whitenization, while the main problem of whitenization is that the techniques of collecting and dealing with data generally do not perforate through to the end of study so that there is no guarantee for the techniques to produce a cover for the connotation of the matter of concern. That is the reason why the difference, if any, between techniques of handling grey information and the methods of the classical information analysis has not been clearly shown. This weakness is also the main reason for some scholars to question the validity of grey systems theory.

\section{Application system of grey systems engineering}

In the physical world, most systems considered in ecological environments, societies, economies, scientific research, etc., are complicated. The complexity of systems creates the appearance of poor information in human understanding of the connotations of the internal matters of objective systems. That is why these systems should be seen as grey. Grey systems theory plays the role of bridging social and natural sciences and helps to quantify abstract systems. The totality of all interdisciplinary studies based on special knowledge of particular disciplines and the fundamental principles and methods of grey systems theory is referred to as grey systems engineering. For instance, when the principles and methods of grey systems are employed to study hydrologic phenomena using poor information, one establishes a specific theoretic system of concepts and methods of hydrology. Such an area of learning has been known as grey hydrology. Currently, some of the main works in grey systems engineering include grey input-output analysis (see [14-16]), grey hydrology (see [37-38]), grey extenics (see [39]), etc. Because the phenomena of poor information always exists with complex systems, grey systems theory can be expected to play its role in a wide range of disciplines. 


\section{Final words}

Although in the past thirty years grey systems theory has grown tremendously, there are still many fundamental problems that await resolution. Because of this, parts of grey systems theory are still evolving in different directions, attracting challenges from various scholars. The purpose of this paper is not about the exploration of the theoretical problems facing grey systems research; instead, it is about presenting the main results of this extremely active area of scientific endeavors so that scholars from other disciplines can join in the effort to further develop the theory into a mature system of knowledge.

\section{References}

[1] Wang L X. Fuzzy systems: Challenges and chance - My experiences and perspectives. ACTA Automatica Sinica, 2001, 27(4): 585-590.

[2] Liu S F, Forrest J, Vallee R. Emergence and development of grey systems theory. Kybernetes: The International Journal of Systems and Cybernetics, 2009, 38(7-8): 1246-1256.

[3] Liu S F, Lin Y. Advances in grey systems research. Berlin: Springer-Verlag, 2010b.

[4] Huang Y P. Practical consideration for grey modeling and its application to image processing. The Journal of Grey Systems, 1996(3): 217-234.

[5] Xu Z X, Wu G. The sliding grey relational method for delineating regions containing oil and gas. The Journal of Grey Systems, 1996(3): 275-282.

[6] Lin Y, Valencia J. Grey analysis of Colombian migration. The Journal of Grey Systems, 2008, 20(1): 5-20.

[7] Liu S F, Lin Y. The role and position of grey systems theory in science development. Journal of Grey Systems, 1997, 4: 351-356.

[8] Li Q X, Lin Y. The basic architecture of grey system theory. Proceedings of the 2010 Conference on Management in China, Lanzhou, China, 2010: 158-163.

[9] Liu S F, Lin Y. Grey systems: Theory and applications. Berlin: Springer-Verlag, 2010a.

[10] Lin Y, Chen M Y, Liu S F. Theory of grey systems: Capturing uncertainties of grey information. Kybernetes: The International Journal of Systems and Cybernetics, 2004, 33(2): 196-218.

[11] Lin Y, Liu S F. Solving problems with incomplete information: A grey systems approach. Advances in Imaging and Electron Physics, published by Elsevier, Oxford, UK, 2006, 141: 77-174.

[12] Wang X Y. Conspectus to philosophy and science. Guangzhou: South China University of Technology Press, 2007.

[13] Deng J L. Elements of grey theory. Wuchang: Press of Huazhong University of Science and Technology, 2002.

[14] Li Q X. Research on the grey input-output analysis and the extensive adjustment of direct consumption coefficient. Nanjing: Nanjing University of Aeronautics and Astronautics, 2007.

[15] Li Q X, Liu S F. The foundation of the grey matrix and the grey input-output analysis. Applied Mathematical Modelling, 2008, 32(3): 267-291.

[16] Li Q X, Liu S F. Some results about grey mathematics. Kybernetes, 2009(3/4): 297-305.

[17] Sunaga T. Theory of interval algebra and its application to numerical analysis. RAAG Memoires, 1958, 2: $29-46$.

[18] Moore R E. Interval arithmetic and automatic error analysis in digital computing. Department of Mathematics, Stanford University, Stanford, CA, 1962.

[19] Liu S F, Lin Y. Grey information: Theory and practical applications. London: Springer, 2006.

[20] Hayes B. A lucid interval. American Scientist, 2003, 91(6): 484.

[21] Dwyer P S. Linear computations. London: Chapman \& Hall, 1951.

[22] Warmus M. Calculus of approximations. Bull Acad Polon Sci, 1956, Cl. III, IV(5): 253-259.

[23] Warmus M. Approximations and inequalities in the calculus of approximations: Classification of approximate numbers. Bull Acad Polon Sci, Ser math, astr et phys, 1961, IX(4): 241-245.

[24] Moore R E. Interval analysis. Englewood Cliffs, NJ: Prentice Hall, 1966.

[25] Alefeld G, Herzberger J. Einführung in die Intervallrechnung. Bibliographisches Institute, Reihe Informatik, 
Band 12, B.I.-Wissenschaftsverlag, Mannheim-Wien-Zürich, 1974.

[26] Kulisch U. Wissenschaftliches Rechnen mit Ergebnisverifikation. Eine Einführung, Vieweg-Verlag, Wiesbaden, 1989.

[27] Nickel K L. Uber die notwendigkeit einer fehlerschranken: Arithmetik fur rechenautomaten. Numerische Mathematrk, 1966, 9(1): 69-79.

[28] Neumaier A. Interval methods for systems of equations. Cambridge: Encyclopedia of Mathematics and Its Applications 37, Cambridge University Press, 1990.

[29] Hansen E R. Interval arithmetic in matrix computations, Part 1. Journal of the Society for Industrial and Applied Mathematics: Series B, Numerical Analysis, 1965, 2(2): 308-320.

[30] Hansen E R. On solving systems of equations using interval arithmetic. Mathematics of Computation, 1968, 22(102): 374-384.

[31] Hansen E R. Topics in interval analysis. London: Oxford University Press, 1969.

[32] Hansen E R. Global optimization using interval analysis. New York: Marcel Dekker, 1992.

[33] Ratschek H, Rokne J G. Computer methods for the range of functions. Ellis Horwood Ser: Math Appl Chichester, UK: Ellis, 1984.

[34] Hansen E R, Walster G W. Global optimization using interval analysis. New York: Marcel Dekker, 2003.

[35] Lin Y, OuYang S C. Irregularities and prediction of major disasters. New York: CRC Press, 2010.

[36] Xiao X P, Song Z M, Li F, et al. The foundation of grey technology and its applications. Beijing: China Science Press, 2005.

[37] Xia J. Identification of a grey parameter model and its application to hydrological mid-long term forecasting. Journal of Huazhong University of Science and Technology, 1994, 22(1): 55-60.

[38] Zhang W S, Wang Y T. Application of the time series decomposable model in medium and long-term hydrologic forecasting. Hydrology, 2001, 21(1): 21-24.

[39] Li Q X. The grey extensible set. Proceedings of International Conference on Machine Learning and Cybernetics, Kunming, China, 2008: 2390-2394. 